\title{
Основные эффекты кислородных центров в оптике $\mathrm{A}^{\text {"I }}$ VI
}

\author{
() Н.К. Морозова ${ }^{1}$, И.И Аббасов ${ }^{2}$ \\ ${ }^{1}$ Национальный исследовательский университет „Московский энергетический институт“, \\ 111250 Москва, Россия \\ ${ }^{2}$ Азербайджанский государственный университет нефти и промышленности, \\ AZ1010 Баку, Азербайджан \\ E-mail: MorozovaNK@mail.ru
}

Поступила в Редакцию 26 декабря 2021 г.

В окончательной редакции 10 января 2022 г.

Принята к публикации 10 января 2022 г.

На основании проведенных исследований в работе показано, что влияние кислорода на оптические свойства кристаллов $\mathrm{A}^{\mathrm{II}} \mathrm{B}^{\mathrm{VI}}$ определяется тремя основными эффектами. Это - образование системы связанных экситонов, сопровождающих присутствие больших концентраций кислорода на дефектах упаковки, длинноволновый сдвиг на сотни мэВ от края поглощения и третий эффект определяется возникновением широкополосного „самоактивированного“ SA-свечения в прикраевой области спектра. В работе введены представления о неравномерном распределении изоэлектронных кислородных центров в объеме кристаллов как вследствие преимущественной сегрегации их на дефектах упаковки, так и в этих слоях. Для анализа экспериментальных данных использовались возможности построения зонных моделей, которые объединяют обширную и разностороннюю информацию о конкретных образцах. Представлена уточненная модель мультизоны $\mathrm{CdS} \cdot \mathrm{O}$ с дефектами упаковки. Описаны условия и возможность совместного или раздельного наблюдения этих особенностей в спектрах разных кристаллов $\mathrm{A}^{\mathrm{II}} \mathrm{B}^{\mathrm{VI}}$ и использования их для создания лазеров.

Ключевые слова: зонная модель, связанные экситоны, край дополнительного поглощения, дефекты упаковки, точечные дефекты, теория антипересекающихся зон, лазерный эффект.

DOI: $10.21883 /$ FTP.2022.05.52350.9793

\section{1. Введение}

В предшествующие годы роль кислорода в оптике кристаллов $\mathrm{A}^{\mathrm{II}} \mathrm{B}^{\mathrm{VI}}$ рассматривалась в основном с точки зрения зависимости от присутствия и концентрации кислорода самоактивированного SA-излучения. Было выявлено также аномальное длинноволновое смещение на сотни мэВ от края дополнительного поглощения кристаллов, в которых предполагалось увеличение содержания кислорода.

Проведенные нами исследования краевого свечения $\mathrm{CdS}, \mathrm{ZnS}$ и $\mathrm{ZnSe}$ обнаружили, кроме того, группу узких полос, обязанных экситонам, связанным на кислородных центрах в слоях дефектов упаковки $\left(B E_{e x}\right)$ [1-7].

При аннигиляции экситонов с увеличением плотности возбуждения наблюдалось вынужденное излучение и лазерный эффект.

В данном сообщении сделана попытка сконцентрировать полученные итоговые результаты на основе более подробного анализа условий и возможности совместного и раздельного наблюдения этих особенностей в спектрах кристаллов $\mathrm{A}^{\mathrm{II}} \mathrm{B}^{\mathrm{VI}}$.

\section{2. Материалы и методики эксперимента}

Рассмотрены в основном результаты исследования кристаллов $\mathrm{CdS}, \mathrm{ZnS}$ и $\mathrm{ZnSe}$, выращенных из газо- вой фазы. Работа базируется на исследовании группы кристаллов с заданным при выращивании отклонением от стехиометрии. При этом реальные монокристаллы $\mathrm{CdS}(\mathrm{ZnS})$ из газовой фазы растут обычно в основной центральной части области гомогенности.

Для селенида цинка рассмотрены образцы, выращенные CVD методом при большом избытке селена. Концентрация кислорода в кристаллах $\sim 10^{20} \mathrm{~cm}^{-3}$, чему способствовала соактивация в условиях роста медью [5].

При исследовании фото- и катодолюминесценции кристаллов использовались различные методики возбуждения с изменением интенсивности возбуждения катодолюминесценции и импульсного возбуждения, методы локальной съемки в растровом электронном микроскопе (РЭМ), а также фотолюминесценция при шаговом изменении на $\sim 30$ мэВ энергии возбуждения в области края фундаментальной абсорбции. Использованы данные спектров поглощения и отражения.

\section{3. Заключительное обсуждение экспериментальных данных}

На рис. 1 основные эффекты, определяющие роль кислорода, представлены на примере $\mathrm{CdS}(\mathrm{O})$ в зонных моделях кристалла с дефектами упаковки. Построение выполнено на основе принципов теории антипересекающихся зон (band anticrossing theory - BAC) [4,8-10]. 

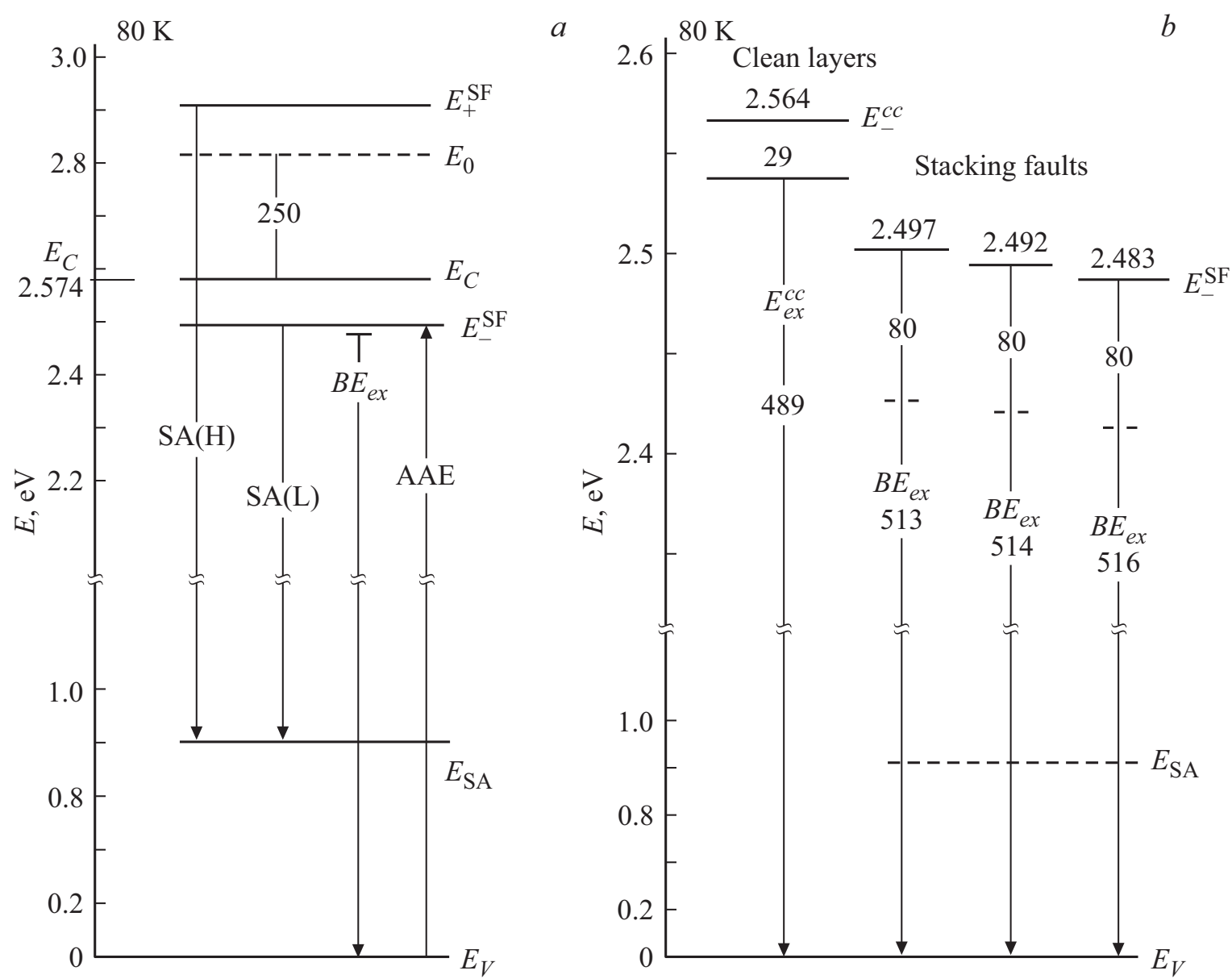

Рис. 1. Зонные модели кристалла $\mathrm{CdS} \cdot \mathrm{O}: a-$ выделены полосы излучения и поглощения, обязанные SA-центрам, $b-$ мультизона того же кристалла на ДУ для спектра зеленого краевого свечения CdS · О. Отмечены глубокие уровни рекомбинации $E_{\mathrm{SA}}$ самоактивированного SA-излучения [2]. Положения уровней $E_{+}^{\mathrm{SF}}$ и $E_{-}^{\mathrm{SF}}$ в слоях ДУ (stacking faults $\mathrm{SF}$ ) даны, полагая, что смещение подзон линейно и соответствует $\sim 90$ мэВ на 1 мол\% растворенного кислорода [1].

Модель базируется на следующих особенностях кислорода в $\left.\mathrm{A}^{\mathrm{II}} \mathrm{B}^{\mathrm{VI}} \quad[1]: 1\right)$ кислород всегда присутствует в соединениях $\mathrm{A}^{\mathrm{II}} \mathrm{B}^{\mathrm{VI}}$, что подтверждено анализами и обосновано термодинамически; 2) компенсация объемного несоответствия кислородных центров $\mathrm{O}_{\mathrm{S}}$ (или кислородных комплексов) решеткой осуществляется за счет образования дефектов упаковки (stacking faults $\mathrm{SF})$; 3) присутствие $\mathrm{O}_{\mathrm{S}}$ в повышенных концентрациях на дефектах упаковки сопровождается изменением в соответствии с теорией ВАС [8-10] зонной структуры кристалла.

Для кристаллов $\mathrm{CdS}(\mathrm{ZnS})$, которые обычно имеют состав, близкий к стехиометрии с небольшим избытком металла, характерны SA-центры. Согласно [1], это комплексы собственных дефектов и кислорода $\left\{\mathrm{O}_{\mathrm{S}} \mathrm{Cd}_{i}^{\bullet} \mathrm{V}_{\mathrm{Cd}}^{/ /}\right\}^{\prime}$ в случае CdS.

В левой части диаграммы рис. $1, a$ выделены полосы самоактивированной люминесценции и поглощения в кристаллах с $\left[\mathrm{O}_{\mathrm{S}}\right] \sim 10^{20} \mathrm{~cm}^{-3}$, обязанные SA-центрам. $\mathrm{B}$ соответствии с теорией ВАС две полосы самоактивированного свечения сульфида кадмия $(\mathrm{SA}(\mathrm{H}) 600$ и $\mathrm{SA}(\mathrm{L}) 720$ нм) определяются двумя переходами на глубокие уровни центров рекомбинации: $E_{-}^{\mathrm{SF}} \rightarrow E_{\mathrm{SA}}$ и $E_{+}^{\mathrm{SF}} \rightarrow E_{\mathrm{SA}}[1]$.

Кислородные SA-центры определяют и второй эффект - край дополнительного поглощения (additional absorption edge - AAE). На рис. 1, $a$ это соответствует переходам $E_{V} \rightarrow E_{-}^{\mathrm{SF}}$. Возможно также поглощение SA-центрами.

Спектр $B E_{e x}$ вынесен отдельно на правую часть диаграммы (рис. $1, b$ ). Диаграмма построена по экспериментальным данным, детально описанным в работе [2]. В светлых слоях $\left[\mathrm{O}_{\mathrm{S}}\right] \sim 2 \cdot 10^{19} \mathrm{~cm}^{-3}$. Полоса излучения чистых слоев $\mathrm{CdS} E_{e x}^{c c}$ при при больших интенсивностях возбуждения определяет, согласно [2], лазерный эффект.

Спектр связанных экситонов $\left(B E_{e x}\right)$ в слоях дефектов упаковки CdS (рис. $1, b$ ) возникает вследствие переходов из подзоны $E_{-}^{\mathrm{SF}}$, созданной SA-центрами, всегда присутствующими в таких кристаллах на дефектах упаковки, в валентную зону $E_{V}$. Набор полос краевого свечения $B E_{e x}$ в CdS·O определяется аннигиляцией экситонов участков кристалла, которые несколько отличаются по 


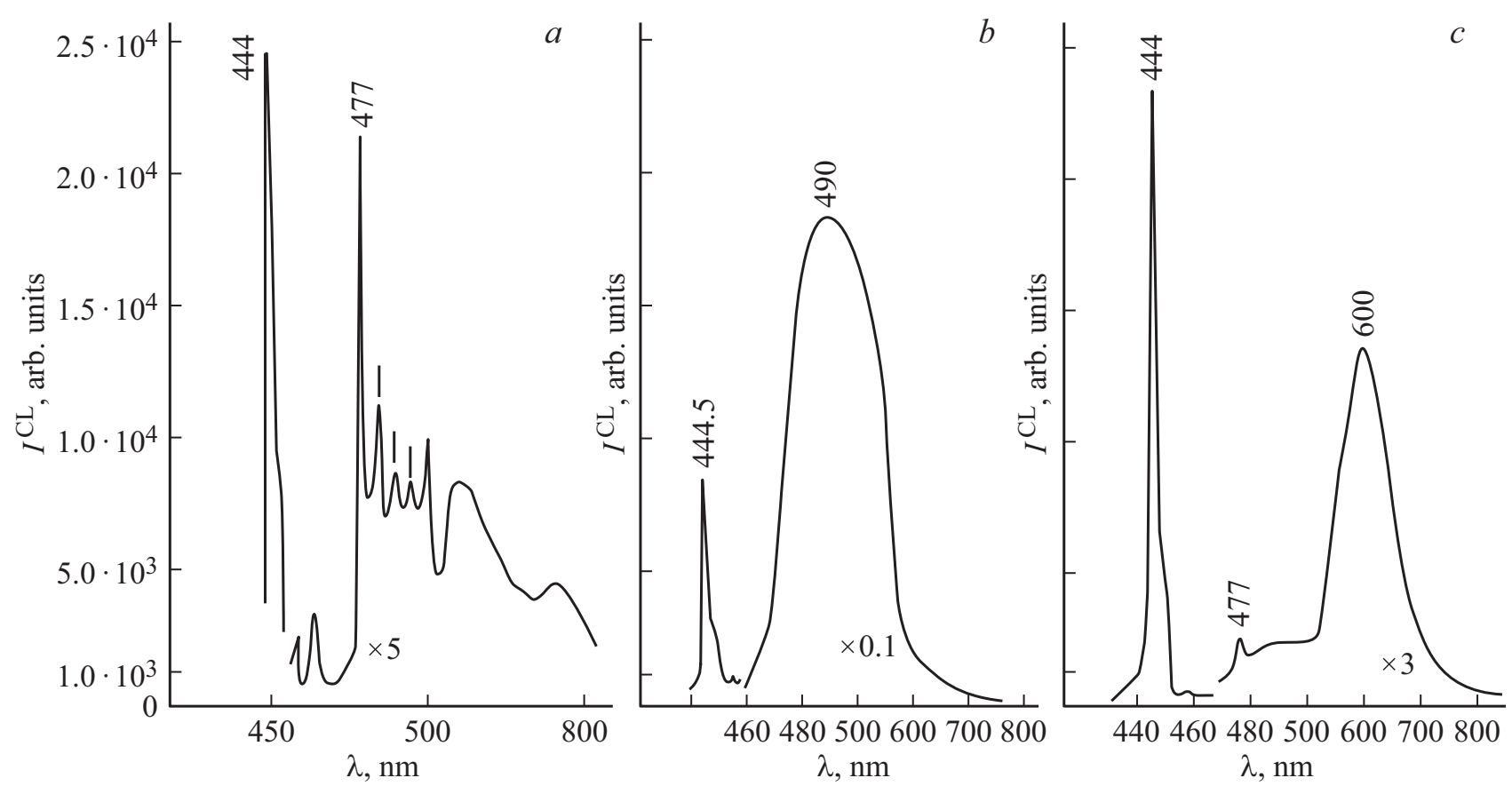

Рис. 2. Изменение спектров КЛ при $80 \mathrm{~K}$ по длине CVD конденсата $(a \rightarrow c)$ : на входе в реактор $(a)$; при уменьшении в газовой фазе давления паров Se и незначительно кислорода $(b \rightarrow c)$ [3].

содержанию в них кислорода. Это соответствует исследованиям в РЭМ слоев ДУ (см. далее).

Как усреднение для всех близко расположенных уровней связанных экситонов $\left(B E_{e x}\right)$ на рис. $1, b$ дано обозначение дна подзоны всего слоя дефекта упаковки $-E_{-}^{\mathrm{SF}}$.

Рассчитанные по спектрам зеленого краевого свечения $\mathrm{CdS} \cdot \mathrm{O}$ величины концентраций кислорода на ДУ выявляют сравнительно неширокий диапазон составов кристаллов, для которых кислородные SA-центры полностью компенсированы собственными точечными дефектами $[11,12]$.

Согласно этим работам, система уровней локализованных экситонов $B E_{e x}$ на дефектах упаковки не изменяется с температурой. Полосы $B E_{e x}$ наблюдались при одних и тех же длинах волн как при 80, так и при $300 \mathrm{~K}[1,2,11,12]$.

В отличие от рассмотренных кристаллов $\mathrm{CdS}$, селенид цинка обладает широкой областью гомогенности со стороны избытка селена [1-3] и может быть получен со значительным избытком селена. Спектр излучения таких кристаллов определяет новые особенности $[1,3,5]$.

На рис. 2 представлены образцы CVD $\mathrm{ZnSeSe}(\mathrm{O})$, выращенные при введении избытка селена и кислорода до 4\% в газовой фазе. Расчет равновесия собственных точечных дефектов в условиях роста показал, что диапазон составов образцов смещен существенно от „области

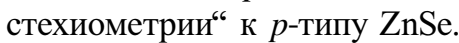

Как видно из рис. 2, $a$, для $\mathrm{ZnSeSe}(\mathrm{O})$ наблюдается группа очень узких ( 20 мэВ $)$ полос с головной линией 477 нм и LO фононными повторами в области 477-490нм. При увеличенной $\left[\mathrm{O}_{\mathrm{S}}\right]$ и селе- на (рис. $2, b)$ все компоненты этого многополосчатого спектра $\mathrm{ZnSeSe}(\mathrm{O})$ усиливаются, перекрываются и дают суммарную широкую полосу с максимумом $\sim 490 \mathrm{Hм}$. Полоса $\sim 490$ нм очень интенсивна и доминирует во всей области спектра рис. $2, b$.

Изменения спектра с уменьшением избытка $\mathrm{Se}$ при росте и обогащении кристаллов цинком показаны на рис. $2(b \rightarrow c)$. При этом полоса 490 нм уменьшается по интенсивности, выделяются отдельные ее компоненты, в частности 477 нм. С избытком Zn в кристаллах появляется, как основное, самоактивированное свечение 600 нм - полоса SA(L).

$\mathrm{C}$ повышением температуры спектральный сдвиг компонент этой группы узких линий отсутствует. Исследование полосы 477 нм при высоких интенсивностях возбуждения показало, что максимум ее не смещается и при увеличении температуры до $300 \mathrm{~K}$, когда полоса доминирует в спектрах $[3,4]$. В спектрах импульсной КЛ при плотности возбуждения $\geq 10^{25} \mathrm{~cm}^{-3} \cdot \mathrm{c}^{-1}$ с усилением интенсивности полосы 477 нм возникает вынужденное излучение и наблюдается лазерный эффект [7].

Уточнение положения уровня $E_{+}^{\mathrm{SF}}$ в зонной модели $\mathrm{ZnSeSe}(\mathrm{O})$ по данным работы [6] позволило определиться с природой этих узких полос. На рис. 3 представлена зонная модель $\mathrm{ZnSeSe}(\mathrm{O})$ с дефектами упаковки по экспериментальным данным этой работы.

Обозначение $E_{-}^{\mathrm{SF}}$ определяет положение дна зоны проводимости в слое дефекта упаковки. Основная полоса 477 нм, согласно рис. $3, b$, соответствует связанному $B E_{e x}$ экситону. Такая интерпретация отличается 

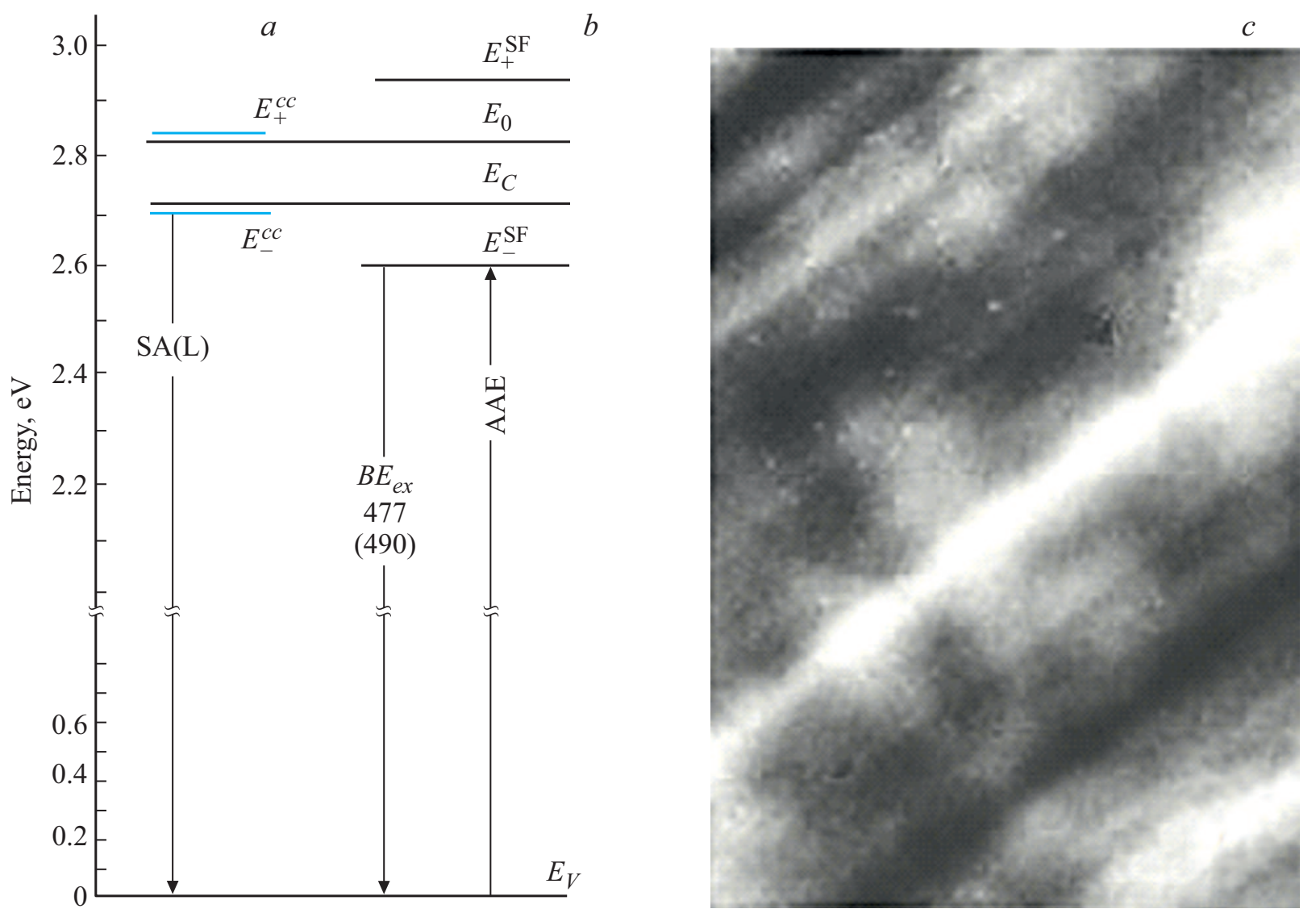

Рис. 3. Мультизона кристалла $\mathrm{ZnSeSe}(\mathrm{O})$ по экспериментальным данным [6]: чистые слои $(a)$, слои дефектов упаковки (b). Края подзон чистых слоев на рис. $3, a$ обозначены как $E_{-}^{c c}\left(E_{+}^{c c}\right)$. Смещение подзон соответствует $\sim 100$ мэВ на 1 мол\% растворенного кислорода в $\mathrm{ZnSe}[1]$. На рис. 3, с представлена микрофотография в РЭМ моноскола $\mathrm{CdS}$ при $\times 5000$ в режиме сотро. Черные участки с повышенным содержанием кислорода выделяют кислород и слои дефектов упаковки.

от описания этих спектров $\mathrm{ZnSeSe}(\mathrm{O})$ ранее в $[1,3,5]$ как $\mathrm{SAL}(\mathrm{II})$.

В относительно неоднородных слоях дефектов упаковки (рис. $3, c$ ) полоса 477 нм относится к участкам с минимальной $\left[\mathrm{O}_{\mathrm{S}}\right]$, в то время как $490 \mathrm{Hм}-\mathrm{\kappa}$ максимально легированным кислородом.

В отличие от рассмотренных кристаллов $\mathrm{CdS}$, на pис. $3, b$ не показаны переходы с излучением или поглощением с участием SA-комплексов. Это определяется составом кристаллов $\mathrm{ZnSeSe}(\mathrm{O})$, в которых при избытке Se отсутствуют точечные дефекты, необходимые для образования SA-комплексов. Широкие полосы самоактивированного SA-свечения $\mathrm{ZnSe} \sim 540$ нм (SA-H) и $\sim 630$ нм (SA-L) в спектрах таких кристаллов $\mathrm{ZnSeSe}(\mathrm{O})$ не наблюдаются. Основной излучательный канал $E_{-}^{\mathrm{SF}} \rightarrow E_{V}$ определяется $B E_{e x}$ экситонами. При больших интенсивностях возбуждения он определяет лазерный эффект [7]. Край дополнительного поглощения таких кристаллов сдвинут в длинноволновую сторону только на величину смещения уровня $E_{-}^{\mathrm{FS}}$ по отношению к $E_{C}$ (рис. $\left.3, b\right)$.

Спектр связанного экситона $B E_{e x}$ как самостоятельный может наблюдаться только для определенных со- ставов $\mathrm{ZnSe}$ с избытком селена, когда отсутствует избыточный цинк $\mathrm{Zn}$ и его комплексы.

Для кристаллов $\mathrm{CdS}(\mathrm{ZnS})$ или $\mathrm{ZnSe}$ с избытком $\mathrm{Zn}$ спектр связанных экситонов $B E_{e x}$ другой, и наблюдать его отдельно невозможно. За присутствие кислорода в кристаллах теперь ответственны SA-центры, которые одновременно определяют широкополосное свечение, дополнительное поглощение и спектр связанных $B E_{e x}$ экситонов.

\section{4. Заключение}

Отметим следующие основные результаты работы.

1. Разделены кислородные центры, ответственные за эффекты в люминесценции и поглощении кристаллов $\mathrm{A}^{\mathrm{II}} \mathrm{B}^{\mathrm{VI}}$. Комплексы кислорода с собственными точечными дефектами $\left\{\mathrm{O}_{\mathrm{S}} \mathrm{A}_{i}^{\bullet} \mathrm{V}_{\mathrm{B}}^{/ /}\right\}^{\prime}$ определяют широкополосное „самоактивированное“" SA-свечение. Они ответственны также за длинноволновый сдвиг края абсорбции на сотни мэВ. При этом неизменно присутствует спектр связанных экситонов на тех же центрах.

2. Показано, что самоактивированные кристаллы, близкие по составу к $p$-типу проводимости и содер- 
жащие повышенные концентрации кислорода, обладают аномальным спектром излучения связанных (локализованных) экситонов, определяющим их оптические свойства.

3. Уточнены зонные модели, описывающие эти эффекты, и природа отдельных полос люминесценции селенида цинка и $\mathrm{CdS}$ с учетом состава и структурных особенностей кристаллов.

4. Описаны условия, позволяющие наблюдать различные виды спектров излучения (поглощения) при изменении собственно-дефектной структуры и состава кристаллов.

5. Сделаны выводы о перспективности использования кристаллов $\mathrm{A}^{\mathrm{II}} \mathrm{B}^{\mathrm{VI}}$ с различной структурой и составом для полупроводниковых лазеров.

\section{Конфликт интересов}

Авторы заявляют, что у них нет конфликта интересов.

\section{Список литературы}

[1] Н.К. Морозова. Новое в оптике соединений II-VI-O: (Новые возможности оптической диагностики монокристаллических систем с дефектами) (LAP LAMBERT Academic Publishing, Riga, Latvia, 2021).

[2] Н.К. Морозова, И.Н. Мирошникова. ФТП, 55 (11), 1068 (2021).

[3] В.В. Блинов. Оптика иентров, обязанных присутствию кислорода и меди в соединениях $A^{I I} B^{V I}$ (на примере $\mathrm{ZnSe}$ ). Канд. дис. (М., МЭИ, 2003).

[4] Д.А. Мидерос. Оптические свойства соединений $A^{I I} B^{V I} c$ изоэлектронной примесью кислорода с позищий теории антипересекающихсязон. Канд. дис. (М., МЭИ, 2008).

[5] Н.К. Морозова, И.Н. Мирошникова. ФТП, $54(1), 59$ (2020).

[6] Н.К. Морозова, И.И. Аббасов, Е.М. Гаврищук, М.А. Мусаев, Дж.И. Гусейнов, А.Дж. Маммадова. ФТП, 56 (1), 80 (2022).

[7] В.И. Олешко, С.С. Вильчинская, Н.К. Морозова. ФТП, $55(5), 403$ (2021).

[8] W. Shan, W. Walukiewicz, J.W. Ager III, K.M. Yu, J. Wu, E.E. Haller, Y. Nabetani, T. Mukawa, Y. Ito, T. Matsumoto. Appl. Phys. Lett., 83 (2), 299 (2003).

[9] M.A. Mayer, Kin Man Yu, E. E. Haller, W. Walukiewicz. J. Appl. Phys., 111 (11), 113 (2012).

[10] W. Walukiewicz, W. Shan, K.M. Yu, M.J. Seong, H. Alawadhi, A.K. Ramdas. Phys. Rev. Lett., 85 (7), 1552 (2000).

[11] N.K. Morozova, A.A. Kanakhin, A.S. Shnitnikov. Semicond., 49 (9), 1134 (2016).

[12] А.А. Канахин. Применение модели антипересекающихся зон в случае высокого легирования кислородом CdS. Канд. дис. (М., МЭИ, 2015).

\section{Main effects of oxygen centers in $A^{\prime \prime} B^{V I}$ optics}

\author{
N.K. Morozova ${ }^{1}$, I.I. Abbasov ${ }^{2}$ \\ ${ }^{1}$ National Research University \\ „Moscow Power Engineering Institute“, \\ 111250 Moscow, Russia \\ ${ }^{2}$ Azerbaijan State Oil and Industry University, \\ AZ1010 Baku, Azerbaijan
}

\begin{abstract}
On the basis of the studies carried out, it is shown in the work that the influence of oxygen on the optical properties of $\mathrm{A}^{\mathrm{II}} \mathrm{B}^{\mathrm{VI}}$ crystals is determined by three main effects. This is the formation of a system of bound excitons accompanying the presence of high oxygen concentrations at stacking faults - a long-wavelength shift by hundreds of meV of the absorption edge, and the third effect is determined by the emergence of broadband „self-activated" SA luminescence in the near-edge region of the spectrum. This work introduces the concept of a non-uniform distribution of isoelectronic oxygen centers in the bulk of crystals, both due to their predominant segregation at stacking faults and in layers. To analyze the optical data, we used the capabilities of the method for constructing band models, which collects extensive and multilateral information about specific samples. The paper describes the conditions and the possibility of joint and separate observation of these features in the spectra of different crystals $\mathrm{A}^{\mathrm{II}} \mathrm{B}^{\mathrm{VI}}$ and using them to create lasers.
\end{abstract}

